\title{
Suspended particle transport through constriction channel with Brownian motion
}

\author{
Hanasaki, Itsuo; Walther, Jens Honore
}

Published in:

Physical Review E (Statistical, Nonlinear, and Soft Matter Physics)

Link to article, DOI:

10.1103/PhysRevE.96.023109

Publication date:

2017

Document Version

Publisher's PDF, also known as Version of record

Link back to DTU Orbit

Citation $(A P A)$ :

Hanasaki, I., \& Walther, J. H. (2017). Suspended particle transport through constriction channel with Brownian motion. Physical Review E (Statistical, Nonlinear, and Soft Matter Physics), 96, [023109].

https://doi.org/10.1103/PhysRevE.96.023109

\section{General rights}

Copyright and moral rights for the publications made accessible in the public portal are retained by the authors and/or other copyright owners and it is a condition of accessing publications that users recognise and abide by the legal requirements associated with these rights.

- Users may download and print one copy of any publication from the public portal for the purpose of private study or research.

- You may not further distribute the material or use it for any profit-making activity or commercial gain

- You may freely distribute the URL identifying the publication in the public portal 


\title{
Suspended particle transport through constriction channel with Brownian motion
}

\author{
Itsuo Hanasaki* \\ Institute of Engineering, Tokyo University of Agriculture and Technology, Naka-cho 2-24-16, Koganei, Tokyo 184-8588, Japan \\ Jens H. Walther \\ Department of Mechanical Engineering, Technical University of Denmark, Building 403, DK-2800 Kgs. Lyngby, Denmark \\ and Swiss Federal Institute of Technology Zürich, Chair of Computational Science, ETH Zentrum, CH-8092 Zürich, Switzerland
}

(Received 13 January 2017; revised manuscript received 12 July 2017; published 31 August 2017)

\begin{abstract}
It is well known that translocation events of a polymer or rod through pores or narrower parts of microand nanochannels have a stochastic nature due to the Brownian motion. However, it is not clear whether the objects of interest need to have a larger size than the entrance to exhibit the deviation from the dynamics of the surrounding fluid. We show by numerical analysis that the particle injection into the narrower part of the channel is affected by thermal fluctuation, where the particles have spherical symmetry and are smaller than the height of the constriction. The Péclet number $(\mathrm{Pe})$ is the order parameter that governs the phenomena, which clarifies the spatio-temporal significance of Brownian motion compared to hydrodynamics. Furthermore, we find that there exists an optimal condition of Pe to attain the highest flow rate of particles relative to the dispersant fluid flow. Our finding is important in science and technology from nanopore DNA sequencers and lab-on-a-chip devices to filtration by porous materials and chromatography.
\end{abstract}

DOI: 10.1103/PhysRevE.96.023109

\section{INTRODUCTION}

When one considers an experiment where a liquid uniformly colored by dye-based ink is injected into syringes, the flow into the needle is expected to have the same color as the rest of the ink. From macroscopic notion without thermal fluctuation, the dye molecules would just follow the streamlines on average. If the suspended particle is actually macroscopic (and shares the same mass density as the surrounding fluid) in place of the dye molecule, the equivalence of particle trajectories with streamlines is justified at low Reynolds number $(\mathrm{Re})$ and Stokes number (Stk) and sufficiently low particle concentration. On the other hand, one may expect that the diffusion of dye molecules due to Brownian motion induced by the thermal fluctuation would always keep the concentration uniform. Both of these conjectures are rather based on the macroscopic continuum notion, where stochasticity of Brownian motion is either ignored by averaging and/or dye molecules are assumed as vanishing size compared to the narrower part of the channel.

There are many situations where small objects subject to Brownian motion in a fluid are introduced to the constriction of microscopic channels, for example, filtration of colloidal particles through porous media [1-3], translocation of DNA molecules through nanopore sequencers [4-17], and lab-on-achip (LOC) devices where analytes are introduced to various domains of nano- and microchannels for analysis [18-25]. It is now well known that the translocation phenomena of polymer molecules through nanopores with a diameter comparable to the monomer size have stochastic nature [5,6]. The smooth entry of the polymers through the nanopores are hindered due to the entropic effect. More specifically, the initiation of a translocation event requires that one end of the polymer arrives at the pore unless the pore has a sufficiently large diameter

\footnotetext{
*Corresponding author: hanasaki@cc.tuat.ac.jp
}

to accommodate the folded translocation. Even if the folded translocation is possible, there is still entropic elasticity of the polymer to resist the folded entrance.

Recent LOC devices also deal with the realm of socalled extended nanospace [26,27]. Injection of a liquid sample suspended with nanoparticles with a diameter of $64 \mathrm{~nm}$ from a microchannel with a height of $6 \mu \mathrm{m}$ into the nanochannel with a height of $410 \mathrm{~nm}$ was found to lead to the difference of particle concentration between the microchannel and nanochannel [26]. This is counterintuitive from the above-mentioned macroscopic viewpoint, and it appears to violate mass conservation. The authors of Ref. [26] argue that the phenomenon originates from the free energy barrier in the nanochannel. However, it is not fully conclusive partly because of the spatio-temporal resolution of the experimental measurement technique. Nevertheless, the number of particles in the observation domain in the nanochannel is considered to be reasonably reliable since the observation in the heightwise direction by total internal reflection fluorescence microscopy fully covers the nanochannel. Therefore, the important question is whether the apparently smaller concentration in the nanochannel is solely caused by the free energy barrier that makes effectively available a volume significantly smaller than that defined by the boundary walls.

There are some related studies where rods instead of a flexible chain exhibit some finite waiting time when entering the constriction [28-30]. Thus, the finite waiting time is not necessarily caused by the many configurations of a flexible chain structure for a given thermodynamic state. However, these existing studies share the feature that the size of a translocated object is larger in some aspect compared to that of constriction. Namely, the entropic barrier of Brownian objects into the constriction in these existing reports is caused by either a configuration within a chain structure or the orientation of an asymmetric shape. In this article, we report that even a simple point particle with perfect spherical symmetry without an inertial effect can exhibit the deviation of time- and 
ensemble-averaged dynamics from continuum flow field when translocated through the constriction part of the channel if the suspended particles are subject to significant Brownian motion.

\section{METHOD}

In this study, we simulate the Brownian motion of suspended particles in stationary fluid flow by the overdamped Langevin equation for a particle subject to a stationary flow field. The flow field of the fluid in the channel is obtained through the lattice Boltzmann method with the multiple-relaxation-time model $[31,32]$. In order to reduce the computational costs, we first obtain the flow field of the fluid and use it for the numerical solution of the Langevin equation for a suspended particle as

$$
\begin{aligned}
\Delta \boldsymbol{r}(t) & \equiv \boldsymbol{r}(t+\Delta t)-\boldsymbol{r}(t) \\
& =\frac{\boldsymbol{f}_{\mathrm{c}}(\boldsymbol{r}(t))}{\xi} \Delta t+\sqrt{\frac{2 k_{\mathrm{B}} T \Delta t}{\xi}} \psi(t)+\boldsymbol{u}(\boldsymbol{r}(t)) \Delta t,
\end{aligned}
$$

where $\Delta \boldsymbol{r}(t)=(\Delta x(t), \Delta y(t), \Delta z(t))$ denotes the displacement of a particle at time $t, \Delta t=2 \mathrm{~ns}$ is the time step of the Brownian dynamics simulation, $\xi$ is the friction coefficient in the Langevin equation, $\boldsymbol{f}_{\mathrm{c}}(\boldsymbol{r})$ is the conservative force between the particle and the channel wall, $k_{\mathrm{B}}$ is the Boltzmann's constant, $T$ is the absolute temperature, $\psi(t)$ is a vector of a normal random number, and $\boldsymbol{u}(\boldsymbol{r}(t))$ is the flow field obtained from the lattice Boltzmann simulation with a grid spacing of $10 \mathrm{~nm}$. The flow velocities at specific real-valued positions of the particles are obtained from linear interpolation of this flow field [33]. The fluid flow affects the dynamics of the particles, but the particles do not affect the flow field in this model. This is consistent with a dilute suspension and reasonable for the regime of low Re and Stk numbers.

The schematic diagram of the constriction channel with flow field is shown in Fig. 1. The flow direction is defined as the $x$ direction and the configuration is uniform in the $y$ direction, whereas the constriction is located by the steps in the $z$ direction. The heights of the channel are $2000 \mathrm{~nm}$ and $h_{\mathrm{n}}$ at the wider and narrower parts, respectively. We

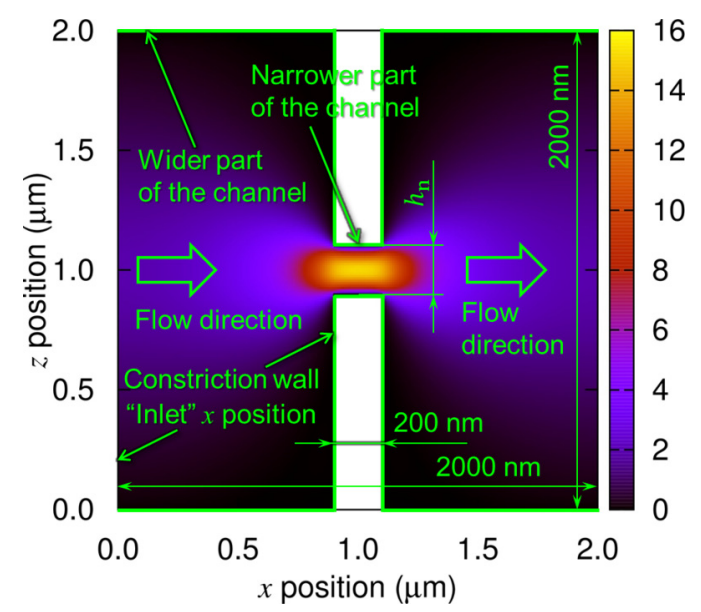

FIG. 1. Schematic diagram of the computational model with the obtained fluid flow field for the case of $h_{\mathrm{n}}=200 \mathrm{~nm}$. The flow speed is colored with a unit scaled by the mean inlet flow velocity at $x=0$. investigate $h_{\mathrm{n}}=200,300$, and $400 \mathrm{~nm}$. The lengths of the narrower part is $200 \mathrm{~nm}$, and the total length in the $x$ direction is $2000 \mathrm{~nm}$. The periodic boundary conditions are assigned in the $x$ and $y$ directions. We model the liquid water at ambient condition for the working fluid, and the relevant physical properties are mass density $\rho=0.998 \times 10^{3} \mathrm{~kg} / \mathrm{m}^{3}$ and viscosity $\eta=1.00 \times 10^{-3} \mathrm{~Pa} \cdot \mathrm{s}$ at $T=293.15 \mathrm{~K}$. The friction coefficient $\xi$ in Eq. (1) is often determined by the diffusion coefficient $D$ by the Einstein relation

$$
\xi=\frac{k_{\mathrm{B}} T}{D} .
$$

The particle-wall interaction is defined by the potential whose functional form is similar to the Weeks-Chandler-Anderson (WCA) type [34]

$$
\begin{gathered}
\boldsymbol{f}_{\mathrm{c}}(\boldsymbol{r})=-\frac{\partial U_{\mathrm{WCA}}(\boldsymbol{r})}{\partial \boldsymbol{r}}, \\
U_{\mathrm{WCA}}(r)= \begin{cases}4 \varepsilon\left[\left(\frac{\sigma}{r}\right)^{12}-\left(\frac{\sigma}{r}\right)^{6}\right]+\varepsilon & \left(0 \leqslant r \leqslant 2^{1 / 6} \sigma\right), \\
0 & \left(r>2^{1 / 6} \sigma\right)\end{cases}
\end{gathered}
$$

where $\boldsymbol{r}$ is replaced, e.g., by $z$ for the top and bottom walls (by $x$ for the side walls), and $\varepsilon=k_{\mathrm{B}} T, \sigma=10^{2} /\left(2^{1 / 6}\right)=89.1 \mathrm{~nm}$, respectively. The walls are perfectly smooth in the plane parallel directions, and hence it is different from the original WCA potential. Therefore, this set of wall potentials is free from direct friction with the particles, whereas a no-slip condition is assigned in the fluid dynamics. $U_{\mathrm{WCA}}(r)$ is shortranged and repulsive. Consistent with the dilute suspension, the particles are assumed not to interact. Although the diffusion coefficient is varied to examine the influence on the dynamics, the geometrical particle size is fixed by $\sigma$. In other words, we directly assign the numerical value of $D$ as an input of Eq. (2) (without the use of Stokes-Einstein equation). It should be noted that the effective size of Brownian nanoparticles in reality has some ambiguity due to, e.g., the electric double layer interaction and molecular softness. We intend to extract essential characteristics in this study rather than quantitatively high precision for a specific system. Therefore, we distinguish the dynamic factor of a diffusion coefficient and geometric factor of particle size compared to the channel height of the narrower part. In particular, we examine the influence of excluded volume between the particle and the walls of the narrower part of the channel by performing another set of simulations defining the extended thickness $R_{\text {excl }}=\sigma / 2$ of the excluded volume in addition to the $\sigma$ for the original potential definition. For the particles, this set of cases is equivalent to experiencing narrower channel height by $2 R_{\text {excl }}=\sigma$ compared to the suspension fluid experiencing $h_{\mathrm{n}}=400 \mathrm{~nm}$.

The particle position in the $z$ direction is initialized when crossing the middle of the wider part of the system in the positive $x$ direction using the uniform random number (cf. the "inlet" $x$ position in Fig. 1). The periodic boundary condition in the $x$ direction can influence the concentration profile in the $x$ direction, but this inlet condition removes the memory of the $z$ position before crossing the inlet in the positive $x$ direction. The initial $z$ positions at the inlet of the recycled particles are uniformly distributed excluding the cutoff distances of 


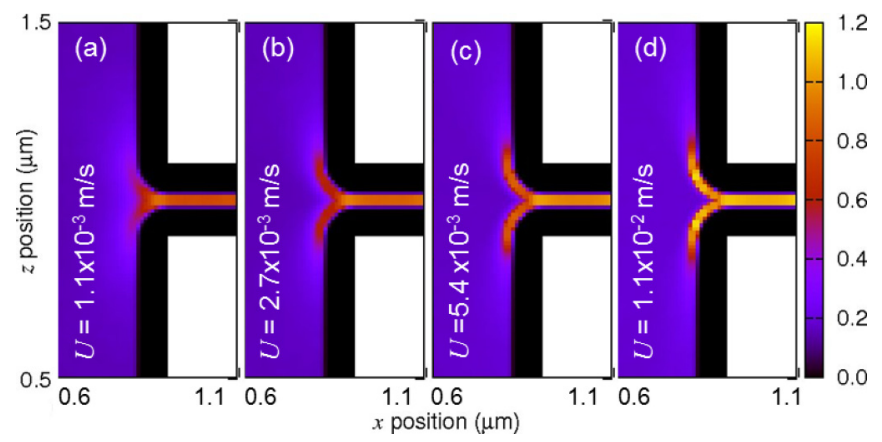

FIG. 2. Particle concentration field with the channel configuration of $h_{\mathrm{n}}=200 \mathrm{~nm}$ for different mean flow velocity $U$ when the diffusion coefficient $D=2.4 \times 10^{-11} \mathrm{~m}^{2} / \mathrm{s}$. The view is zoomed up, and the coordinate values correspond to Fig. 1. The unit of color bars is arbitrary due to the absence of interparticle interaction and no influence of particles on the fluid velocity field in the model.

$2^{1 / 6} \sigma$ from the top and bottom walls, which is necessary for the numerical stability. The particle dynamics is simulated by Eq. (1) for 100 particles for a sampling duration of 10 flow through times after the particle dynamics has reached the steady state in the sense of conservation of particle flow rate between the narrower and wider part of the channel.

\section{RESULTS AND DISCUSSION}

Distributions of particle concentration around the entrance of the narrower part of the channel is shown in Figures 2 to 5. Four cases under different conditions of fluid flow velocities $U$ are shown in each of these figures with a constant diffusion coefficient of $D=4.8 \times 10^{-12} \mathrm{~m}^{2} / \mathrm{s}$, respectively. The velocity $U$ is based on the mean fluid flow velocity in the narrower part of the channel. These figures are based on four types of channel configurations at the narrower part; Fig. 2 corresponds to the cases of $h_{\mathrm{n}}=200 \mathrm{~nm}$, Fig. 3 corresponds to the cases of $h_{\mathrm{n}}=300 \mathrm{~nm}$, Fig. 4 corresponds to the cases of $h_{\mathrm{n}}=400$ $\mathrm{nm}$, and Fig. 5 corresponds to the cases of $h_{\mathrm{n}}=400 \mathrm{~nm}$ with $R_{\text {excl }}=\sigma / 2$. The particle concentrations are roughly uniform in most of the wider part of the channel. The subdomain of zero concentration in the vicinity of the the walls inside the channel

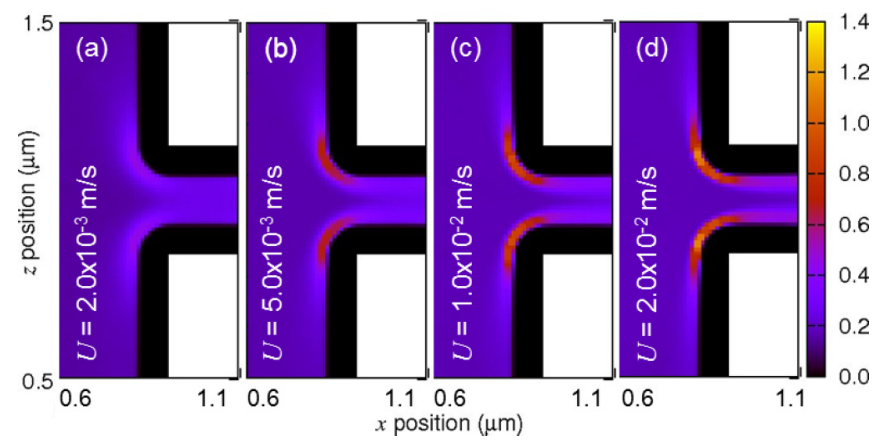

FIG. 3. Particle concentration field with the channel configuration of $h_{\mathrm{n}}=300 \mathrm{~nm}$ for different mean flow velocity $U$ when the diffusion coefficient $D=2.4 \times 10^{-11} \mathrm{~m}^{2} / \mathrm{s}$. The unit of color bars is arbitrary due to the absence of interparticle interaction and no influence of particles on the fluid velocity field in the model.

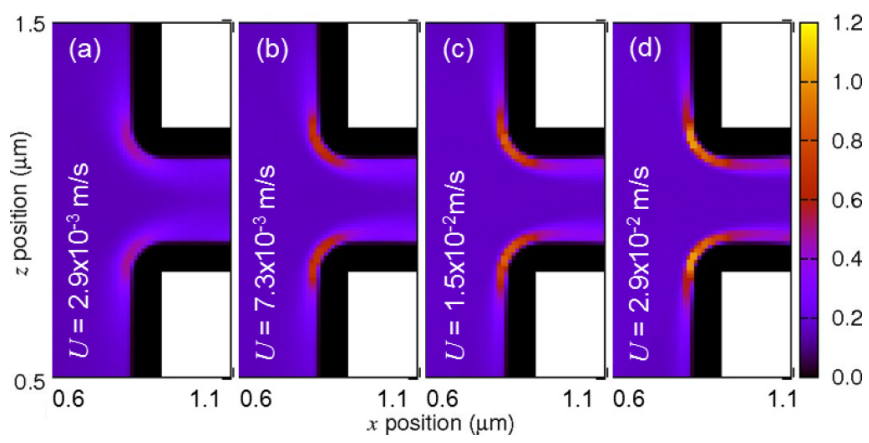

FIG. 4. Particle concentration field with the channel configuration of $h_{\mathrm{n}}=400 \mathrm{~nm}$ for different mean flow velocity $U$ when the diffusion coefficient $D=2.4 \times 10^{-11} \mathrm{~m}^{2} / \mathrm{s}$. The unit of color bars is arbitrary due to the absence of interparticle interaction and no influence of particles on the fluid velocity field in the model.

corresponds to the excluded volume due to the soft repulsive interaction between the particle and walls. The important feature in these figures is that the particle concentration significantly increases at the entrance of the constriction. More specifically, the particle concentration is significantly higher just before entering the narrower part of the channel. Thus, the net distribution of particles is nonuniform in the system.

When $h_{\mathrm{n}}=200 \mathrm{~nm}$ and $D=2.4 \times 10^{-11} \mathrm{~m}^{2} / \mathrm{s}$ (Fig. 2), the particles are concentrated at the center in the $z$ direction in the narrower part of the channel. The particle concentration is also higher in the narrower part compared to the wider part. The increase of fluid flow velocity condition $U$ causes the higher concentration when entering the narrower part of the channel. When $h_{\mathrm{n}}>200 \mathrm{~nm}$ (Figs. 3 and 4), the higher particle concentration is more clearly located at the entrance of the constriction part and/or at the edges in the $z$ direction inside the narrower part of the channel. The comparison of difference in the interaction between the particles and the walls inside the narrower part of the channel under the condition of $h_{\mathrm{n}}=400 \mathrm{~nm}$ (Fig. 4 with Fig. 5) shows that the larger excluded volume (Fig. 5) leads to more pronounced nonuniformity of the particle concentrations.

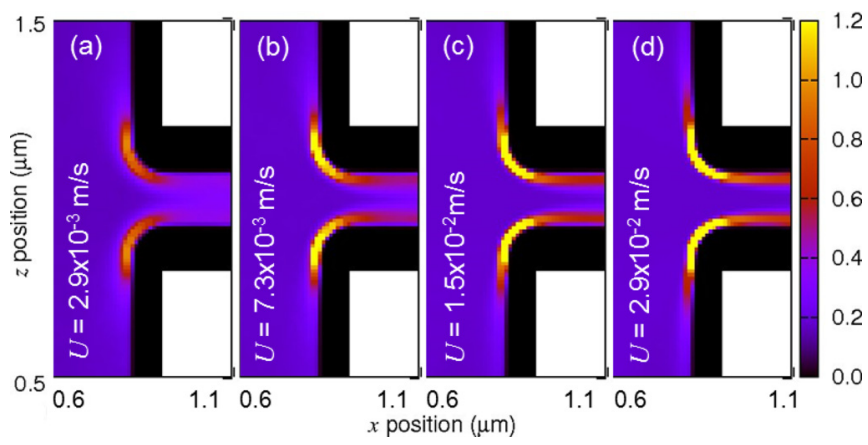

FIG. 5. Particle concentration field with the channel configuration of $h_{\mathrm{n}}=400 \mathrm{~nm}$ and larger excluded volume effect of $R_{\text {excl }}=\sigma / 2$ at the narrower part of the channel, for different mean flow velocity $U$ when the diffusion coefficient $D=2.4 \times 10^{-11} \mathrm{~m}^{2} / \mathrm{s}$. The unit of color bars is arbitrary due to the absence of interparticle interaction and no influence of particles on the fluid velocity field in the model. 


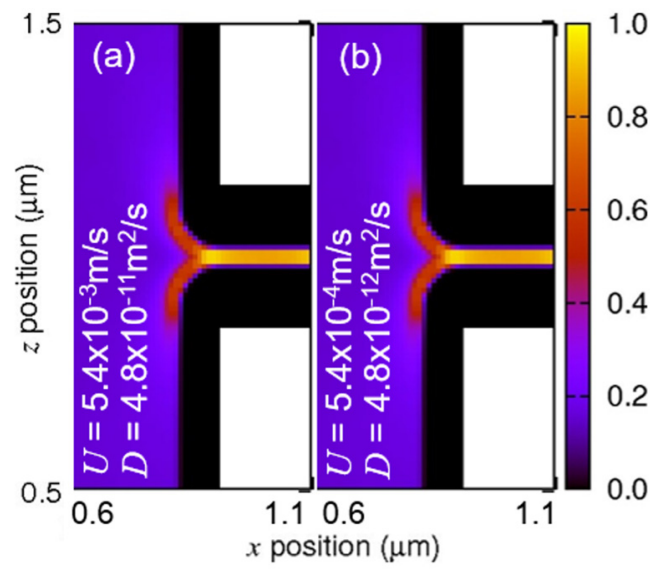

FIG. 6. Particle concentration field with the channel configuration of $h_{\mathrm{n}}=200 \mathrm{~nm}$ for different combinations of velocity $U$ and diffusion coefficient $D$ that corresponds to the same Péclet number $\mathrm{Pe}=22.5$. The view is zoomed up, and the coordinate values correspond to Fig. 1. The unit of color bars is arbitrary due to the absence of interparticle interaction and no influence of particles on the fluid velocity field in the model.

The relative importance of advection to diffusion in continuum fluid dynamics is often expressed in terms of the Péclet number $\mathrm{Pe}=U L / D$, where $U, L$, and $D$ are the representative flow velocity, length, and diffusion coefficient of the system, respectively. Let us define $U$ as the abovementioned mean fluid velocity in the narrower part of the channel, $L$ the height of narrower part of the channel, and $D$ the diffusion coefficient of the suspended particles. Then the dimensionless displacement, time step, and velocity are defined as $\Delta \boldsymbol{r}^{*}=\Delta \boldsymbol{r} / L, \Delta t^{*}=U \Delta t / L$, and $\boldsymbol{u}^{*}=\boldsymbol{u} / U$, respectively. The equation (1) is nondimensionalized using Pe as

$\Delta \boldsymbol{r}^{*}\left(t^{*}\right)=\mathrm{Pe}^{-1} \boldsymbol{f}_{\mathrm{c}}^{*}\left(\boldsymbol{r}^{*}\right) \Delta t^{*}+\left(2 \mathrm{Pe}^{-1} \Delta t^{*}\right)^{1 / 2} \boldsymbol{\psi}(t)+\boldsymbol{u}^{*}\left(\boldsymbol{r}^{*}\right) \Delta t^{*}$.

This equation indicates that the dynamics is governed by the Pe. The increase of Pe results in the more macroscopic conditions, and sufficiently smaller Pe corresponds to the typical nano- and microfluidic conditions. For example, Fig. 6 shows that the variation of both $U$ and $D$ by 10 times corresponding to the same $\mathrm{Pe}=22.5$ results in the same concentration distribution.

The spatial peak values or their locations are likely to be more sensitive to the specifications of channel configurations compared to the related mean values. In order to discuss more robust properties, we then focus on the particle concentrations in the narrower part of the channel. Namely, we consider the following quantity:

$$
C_{\mathrm{n}, \mathrm{p}}^{*}=\frac{\left\langle C^{*}\right\rangle_{\mathrm{n}, \mathrm{p}}}{C_{\mathrm{a}}^{*}},
$$

defined by the ratio of ensemble- and time-averaged particle concentration $\left\langle C^{*}\right\rangle_{\mathrm{n}, \mathrm{p}}$ inside the narrower part of the channel divided by the overall average particle concentration $C_{\mathrm{a}}^{*}$ that corresponds to the value when the particle concentration is completely uniform in the whole simulation domain. Figure 7 shows $C_{\mathrm{n}, \mathrm{p}}^{*}$ as a function of Pe for the different channel configurations and particle-wall interactions. The present results clearly indicate that the $C_{\mathrm{n}, \mathrm{p}}^{*}$ is rather insensitive to the difference of channel configurations and particle-wall interactions. The common feature is that there is a peak value for $C_{\mathrm{n}, \mathrm{p}}^{*}$ as a function of Pe. $C_{\mathrm{n}, \mathrm{p}}^{*}$ decreases with decreasing Pe below certain values of Pe. In the actual experiments, large values of Pe are hard to attain due to the limited speed of fluid flows for smaller channels in general. On the other hand, smaller values of $C_{\mathrm{n}, \mathrm{p}}^{*}$ in the nanochannel with lower pressure difference, i.e., lower Pe with fixed $D$, is experimentally observed as well [26]. In the experimental work of Ref. [26], the order of flow velocity was $10^{-3} \mathrm{~m} / \mathrm{s}$ (hundreds of $\mathrm{nm}$ per $260 \mu \mathrm{s}$ ), the height of the nanochannel was $410 \mathrm{~nm}$, and diffusion coefficient was in the order of $10^{-12} \mathrm{~m}^{2} / \mathrm{s}$, which yields Pe from ca. $10^{2}$ to $10^{3}$. Thus, there is a clear link between our finding and the experimental report on the basic trend of concentration in the narrower part of the channel as a function of Pe.

Next, we examine the net speed of particle flow inside the narrower part of the channel, i.e., after the passing the constriction entrance. Since our model definition is based on sufficiently low Stk (and Re), the particle velocity at every local position simply follows the fluid flow velocity there except for the Brownian displacements. Nevertheless, the ensemble average value without the distinction of $z$ positions can be different under different conditions due to the differences in the particle distributions. Therefore, we define the dimensionless particle velocity inside the narrower part of the channel as

$$
u_{\mathrm{n}, \mathrm{p}}^{*}=\frac{\langle u\rangle_{\mathrm{n}, \mathrm{p}}}{U},
$$

where $\langle u\rangle_{\mathrm{n}, \mathrm{p}}$ is the ensemble average value of the particle velocities in the $x$ direction irrespective of the $z$ positions, and $U$ is the $z$-averaged fluid flow velocity in the narrower part of the channel as already defined in the above discussion. Thus, $u_{\mathrm{n}, \mathrm{p}}^{*}$ indicates the deviation of net particle flow velocity in the narrower part of the channel from the fluid. Figure 8 shows the overall trend that the particle flow velocity inside the narrower part of the channel is always higher than the fluid within the range investigated here. This is because the excluded volume effect renders the nonuniform distribution of particles in the $z$ direction. More specifically, particles tend to be located away from the wall due to the excluded volume effect. Therefore, the larger excluded volume interaction between the particles and the narrower part of the channel [Fig. 8(d)] results in the higher $u_{\mathrm{n}, \mathrm{p}}^{*}$ compared to the cases of smaller excluded volume [Fig. 8(c)]. When the excluded volume interaction is kept constant [Figs. 8(a)-8(c)], the larger $h_{\mathrm{n}}$ leads to lower $u_{\mathrm{n}, \mathrm{p}}^{*}$ because of the relative fraction of the $z$ range that the excluded volume effect takes place. Furthermore, there exists the Pe dependence of $u_{\mathrm{n}, \mathrm{p}}^{*}$ shared among different channel configurations. The larger Pe leads to the lower $u_{\mathrm{n}, \mathrm{p}}^{*}$ because the particles tend to be introduced into the narrower part of the channel without getting centered under the conditions of larger Pe. This is qualitatively confirmed from the visual inspection of Figs. 2 to 5. The cases of smallest $h_{\mathrm{n}}$ therefore exhibit the saturation of Pe dependence on $u_{\mathrm{n}, \mathrm{p}}^{*}$ at sufficiently low Pe, and the asymptotic value is ca. 1.5 , corresponding to the centered location of particles in the $z$ directions. 

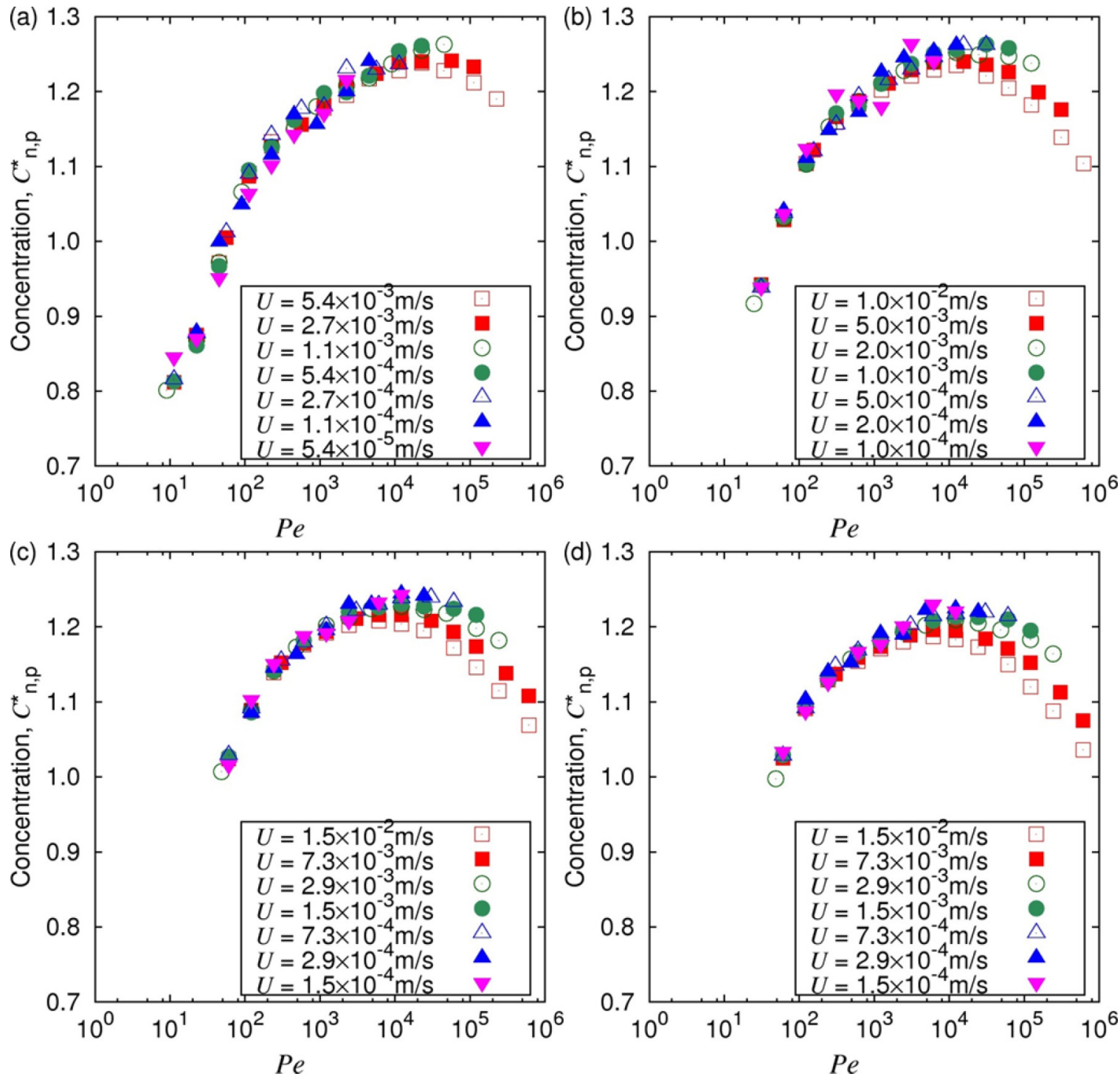

FIG. 7. Dimensionless particle concentration $C_{\mathrm{n}, \mathrm{p}}^{*}$ in the narrower part of the channel as a function of the Péclet number Pe: (a) to (c) correspond to the conditions of $h_{\mathrm{n}}=200,300$, and $400 \mathrm{~nm}$, and (d) corresponds to the condition of $h_{\mathrm{n}}=400 \mathrm{~nm}$ with larger excluded volume effect of $R_{\text {excl }}=\sigma / 2$ at the narrower part of the channel.

From the viewpoint of engineering or experimental applications, the efficiency of particle transport is of great concern. Therefore, we define the dimensionless particle flow rate as

$$
Q_{\mathrm{p}}^{*}=\frac{1}{C_{\mathrm{a}} U h_{\mathrm{n}}}\left\langle\int_{z} C_{\mathrm{n}, \mathrm{p}}(z) u_{\mathrm{n}, \mathrm{p}}(z) d z\right\rangle_{x \in \Omega_{\mathrm{nrw}}},
$$

where the particle flow rate extracted inside the narrower part of the channel (i.e., $x \in \Omega_{\text {nrw }}$ ) is nondimensionalized by the nominal flow rate defined by the above-mentioned overall average of the particle concentration in the whole domain and the average fluid flow velocity. Thus, $Q_{\mathrm{p}}^{*}$ indicates the deviation of particle flow rate compared to the situation where the particle concentrations are uniform throughout the whole simulation domain. Figure 9 shows the dimensionless particle flow rate $Q_{\mathrm{p}}^{*}$ as a function of Pe. There is a common feature that there exists the optimum value of Pe to obtain the highest efficiency of particle transport with respect to the driving of suspension flow. The lower Pe and the higher Pe from the optimum value lead to the decrease of particle flow rate. This overall trend is qualitatively similar to the particle concentration inside the narrower part of the channel (Fig. 7). However, the optimum condition of Pe to obtain highest $Q_{\mathrm{p}}^{*}$ is slightly different from Pe to obtain the highest $C_{\mathrm{n}, \mathrm{p}}^{*}$, due to the Pe dependence of mean particle flow velocity $u_{\mathrm{n}, \mathrm{p}}^{*}$ inside the narrower part of the channel. There is a slight yet significant dependence of $Q_{\mathrm{p}}^{*}$ on $h_{\mathrm{h}}$ that $Q_{\mathrm{p}}^{*}$ is larger for smaller $h_{\mathrm{n}}$ [cf. Figs. 9(a)-9(c)] and larger excluded volume interaction between particles and the walls [cf. Figs. 9(c) and 9(d)]. This dependence corresponds to the particle flow velocity $u_{\mathrm{n}, \mathrm{p}}^{*}$ inside the narrower part of the channel (Fig. 8).

We have shown that there is a nonmonotonic dependence of the particle transport efficiency $Q_{\mathrm{p}}^{*}$ through constriction channel system and the particle concentration $C_{\mathrm{n}, \mathrm{p}}^{*}$ as well as the particle flow velocity $u_{\mathrm{n}, \mathrm{p}}^{*}$ in the narrower part of the channel. The dominating order parameter for the deviation of 

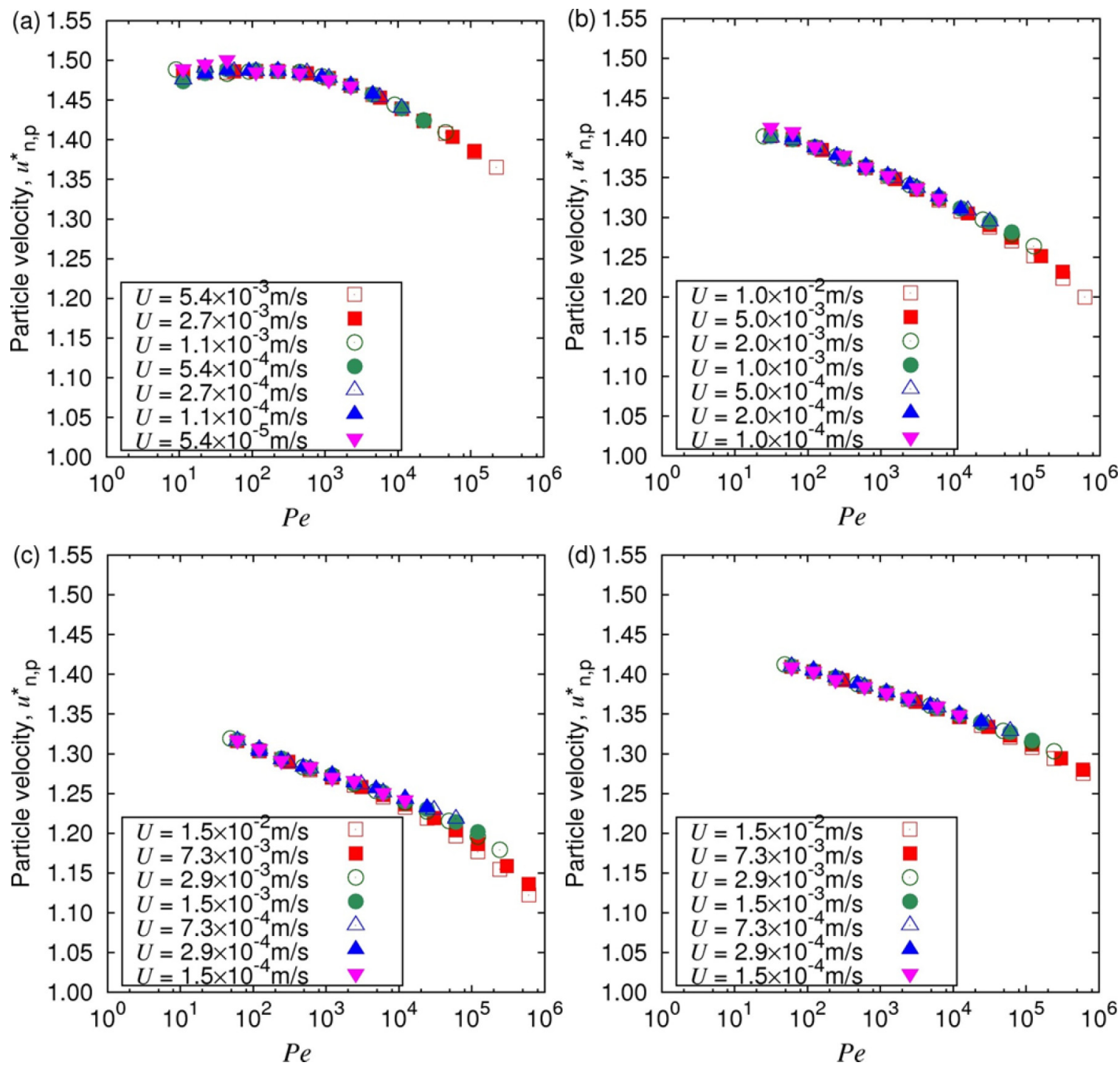

FIG. 8. Dimensionless particle velocity $u_{\mathrm{n}, \mathrm{p}}^{*}$ in the narrower part of the channel as a function of the Péclet number Pe: (a) to (c) correspond to the conditions of $h_{\mathrm{n}}=200,300$, and $400 \mathrm{~nm}$, and (d) corresponds to the condition of $h_{\mathrm{n}}=400 \mathrm{~nm}$ with larger excluded volume effect of $R_{\text {excl }}=\sigma / 2$ at the narrower part of the channel.

net dynamics of suspended particles from the surrounding fluid is determined as Pe. The Brownian motion plays a subtle role in the injection of particles into the narrower part of the channel at the constriction. Particles are accumulated just at the upstream of the entrance of constriction. Nevertheless, the particles can enter the narrower part of the channel since they are smaller than the constriction. The rate of delivery and translocation of particles there determines the concentration field. It leads to the nonmonotonic dependence of particle concentration on $\mathrm{Pe}$ in the vicinity of the constriction in the wider part of the channel and the overall trend of particle concentration inside the narrower part of the channel as shown in Fig. 7. The particle flow rate $Q_{\mathrm{p}}^{*}$ depends on both $C_{\mathrm{n}, \mathrm{p}}^{*}$ and $u_{\mathrm{n}, \mathrm{p}}^{*}$ by construction, and both of the latter depend on Pe. The size of particle-excluded volume compared to the channel height of the narrower part affects the dependence of $u_{\mathrm{n}, \mathrm{p}}^{*}$ and $C_{\mathrm{n}, \mathrm{p}}^{*}$ on Pe. This is related to the nonuniformity in the distributions of particle concentration in the vicinity of the edges of the constriction as shown in Figs. 2 to 5. The particle concentrations at the centers of the narrower part of the channels for $h_{\mathrm{n}}>200 \mathrm{~nm}$ do not exhibit substantial difference from their corresponding wider parts of the channels. It is therefore an interfacial phenomenon. It should be noted here that the interactions between the particles and the channel walls in terms of conservative force field are highly dependent on the solvent specifications from $\mathrm{pH}$ to ion concentrations. Furthermore, it is nontrivial to determine the surface or volume of nanoscale suspended objects including the solute molecules, which is sometimes manifested, e.g., by the discrepancy of hydrodynamic radius and radius of gyration. Therefore, it is important to first consider the simplest kinds of models to discuss the role of Brownian motion in the suspended particle transport through constriction channel.

If the particles have vanishing size or excluded volume and no Brownian motion, the particles just follow the streamlines. The incompressibility of the fluid and the upstream uniform distribution of particles will ensure a constant particle concentration throughout the system. The size of the particles is characterized by $\sigma \sim 10^{-7} \mathrm{~m}$. The ranges of diffusion 

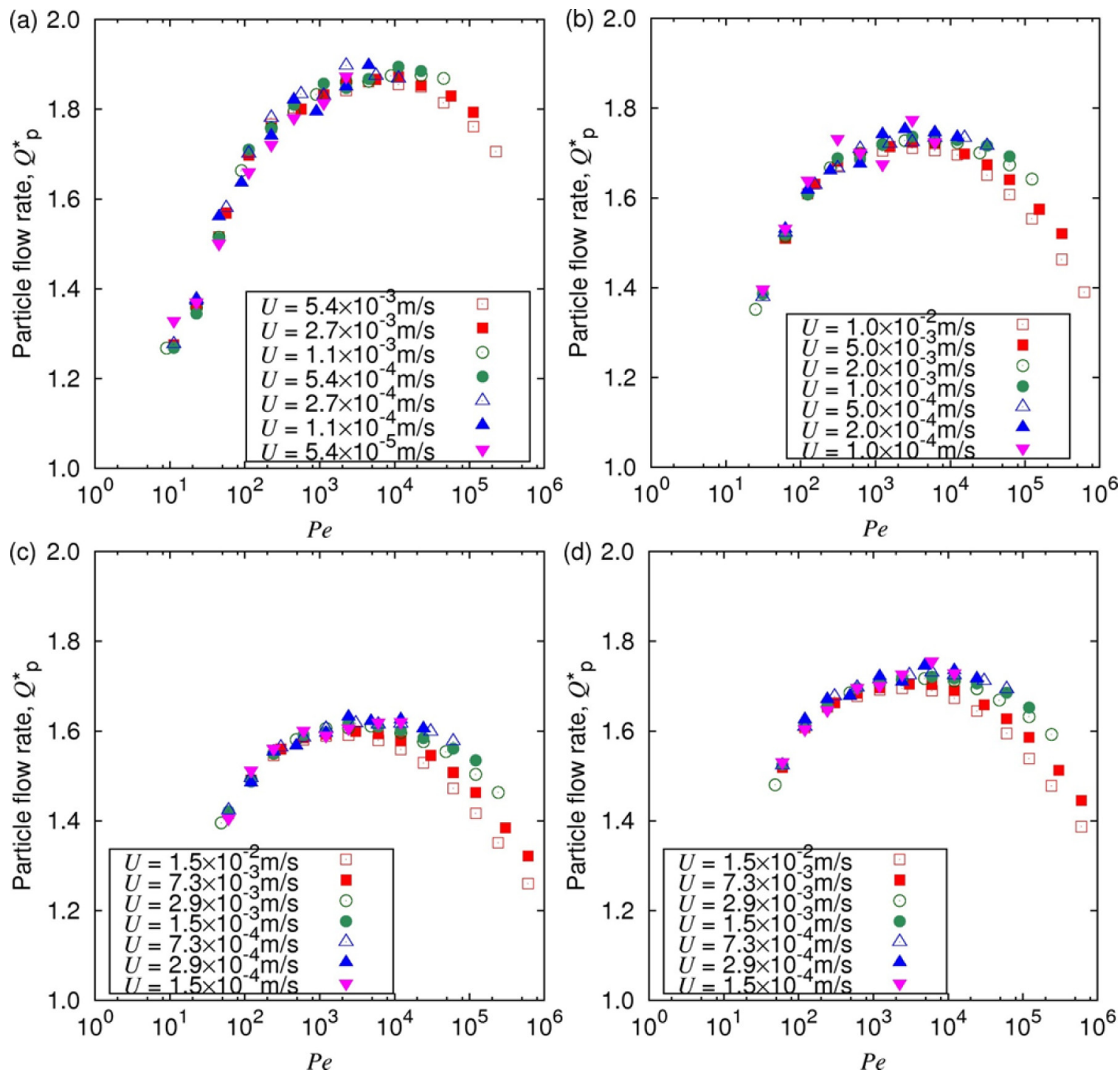

FIG. 9. Dimensionless particle flow rate $Q_{\mathrm{p}}^{*}$ defined in Eq. 8 as a function of the Péclet number Pe: (a) to (c) correspond to the conditions of $h_{\mathrm{n}}=200,300$, and $400 \mathrm{~nm}$, and (d) corresponds to the condition of $h_{\mathrm{n}}=400 \mathrm{~nm}$ with larger excluded volume effect of $R_{\text {excl }}=\sigma / 2$ at the narrower part of the channel.

coefficient $D$ and the velocity $U$ are $D \sim 10^{-15}-10^{-12} \mathrm{~m}^{2} / \mathrm{s}$ and $U \sim 10^{-5}-10^{-2} \mathrm{~m} / \mathrm{s}$, respectively. The time scale $\tau_{\mathrm{B}}$ characterized by the particle size and the Brownian motion is $\tau_{\mathrm{B}} \equiv \sigma^{2} / D \sim 10^{-2}-10^{1} \mathrm{~s}$. The time scale $\tau_{\mathrm{H}}$ characterized by the particle size and the typical or mean fluid velocity is $\tau_{\mathrm{H}} \equiv \sigma / U \sim 10^{-5}-10^{-2} \mathrm{~s}$. Apparently $\tau_{\mathrm{H}}$ is typically smaller than $\tau_{\mathrm{B}}$, but $\tau_{\mathrm{H}} / \tau_{\mathrm{B}} \sim 1$ when the representative fluid velocity is defined as the value close to the wall in the wider part of the channel, which corresponds to the location around the peak concentration of the particles at the entrance of constriction. One of the necessary conditions to observe the deviation of particle transport from the surrounding fluid is considered to be the comparable time scale between the Brownian motion and the hydrodynamic flow in the entry of a particle into the constriction. The dependence of the particle flow rate $Q_{\mathrm{P}}^{*}$ on $\mathrm{Pe}$ is qualitatively interpreted by two competing factors. In the regime of sufficiently low Pe, more intensive Brownian motion hinders the smooth entrance of the particles into the constriction. The particles can often get out of the streamlines that lead to the narrower part of the channel. This is related to the entropic effect in the translocation of a polymer or rod through nanopores. However, it makes a stark difference in that the particles with spherical symmetry have a smaller size compared to the constriction in this study. On the other hand, in the regime of sufficiently high Pe, the particles with significant flow velocities are transported into the narrower part of the channel simply because of the relative characteristic time scale of $U$ to $D$. However, particles in the vicinity of the walls have sufficiently low velocity to be affected by the Brownian motion. The streamlines of these particles are away from the central part of the entrance of constriction, which corresponds to relatively slower transport of the particles into the constriction. When the locations of these particles close to the walls are affected by the Brownian motion, they are transported to the streamlines that are close to the center of narrower part of the channel. This process can enhance the entry of particles into the constriction. Thus, the Brownian motion can affect the transport efficiency of the particles 
through constriction channel in the opposite manner depending on Pe.

\section{CONCLUSIONS}

We have shown that the departure of net particle transport from surrounding fluid emerges at low Reynolds and Stokes numbers without inertial effect. The Péclet number $(\mathrm{Pe})$ is the order parameter that dominates the phenomena. Even when the particle has a smaller size compared to the constriction channel, the stochastic nature of particle dynamics affects the transport efficiency. For sufficiently low Pe, the particle transport through constriction is slowed and the particle concentration in the narrower part of the channel is decreased as Pe is decreased. On the other hand, the slowing of particle transport with increasing Pe is also observed for sufficiently high Pe. We have found that there exists the condition of Pe that maximizes the particle transport relative to the fluid transport. Thus, Brownian motion plays a subtle key role in the transport through the micro- and nanochannels with constriction. We have revealed the phenomena within the finite range of Reynolds number, but this is of great importance in the design of various systems ranging from lab-on-a-chip devices and nanopore DNA sequencers to porous materials to be used for chromatography or any filtration. The diffusion coefficient of particles in fluid is mainly determined by the effective size of particle and viscosity of a fluid under a specific thermodynamic state and channel configuration. The suspension fluid flow velocity might be increased for some systems where a sufficient driving force is available, e.g., in the industrial context of filtration through membranes. Therefore, this nonmonotonic dependence of transport efficiency on $\mathrm{Pe}$ is important in the design of functionality.

\section{ACKNOWLEDGMENT}

This work was partly supported by the Japan Society for the Promotion of Science (JSPS) through a Grant-in-Aid for Young Scientists (A), No. 26709008.
[1] P. de Anna, T. Le Borgne, M. Dentz, A. M. Tartakovsky, D. Bolster, and P. Davy, Phys. Rev. Lett. 110, 184502 (2013).

[2] H. F. Lecoanet, J. Bottero, and M. R. Wiesner, Environ. Sci. Technol. 38, 5164 (2004).

[3] A. M. El Badawy, A. A. Hassana, K. G. Scheckel, M. T. Suidan, and T. M. Tolaymat, Environ. Sci. Technol. 47, 4039 (2013).

[4] F. Detcheverry and L. Bocquet, Phys. Rev. Lett. 109, 024501 (2012).

[5] V. V. Palyulin, T. Ala-Nissila, and R. Metzler, Soft Matter 10, 9016 (2014).

[6] D. Panja, G. T. Barkema, and A. B. Kolomeisky, J. Phys.: Condens. Matter 25, 413101 (2013).

[7] H. Yong, H. Zhang, Y. Xie, and H. Yang, Soft Matter 9, 3565 (2013).

[8] K. Yang, A. Vishnyakov, and A. V. Neimark, J. Phys. Chem. B 117, 3648 (2013).

[9] P. Rowghanian and A. Y. Grosberg, J. Phys. Chem. B 115, 14127 (2011).

[10] K. Luo, T. Ala-Nissila, S. C. Ying, and R. Metzler, Europhys. Lett. 88, 68006 (2009).

[11] T. Saito and T. Sakaue, Phys. Rev. E 85, 061803 (2012).

[12] J. Guo, X. Li, Y. Liu, and H. Liang, J. Chem. Phys. 134, 134906 (2011).

[13] T. Sakaue, Phys. Rev. E 81, 041808 (2010).

[14] A. P. Markesteijn, O. B. Usta, I. Ali, A. C. Balazs, and J. M. Yeomans, Soft Matter 5, 4575 (2009).

[15] M. Fyta, S. Melchionna, S. Succi, and E. Kaxiras, Phys. Rev. E 78, 036704 (2008).

[16] J. P. Hernandez-Ortiz, M. Chopra, S. Geier, and J. J. de Pablo, J. Chem. Phys. 131, 044904 (2009).

[17] J. K. Rosenstein, M. Wanunu, C. A. Merchant, M. Drnic, and K. L. Shepard, Nat. Meth. 9, 487 (2012).

[18] P. Arosio, K. Hu, F. A. Aprile, T. Muller, and T. P. J. Knowles, Anal. Chem. 88, 3488 (2016).
[19] E. K. Sackmann, A. L. Fulton, and D. J. Beebe, Nature (London) 507, 181 (2014).

[20] K. M. Warren, J. N. Mpagazehe, P. R. LeDuc, and C. F. Higgs III, Lab Chip 16, 593 (2016).

[21] R. Vogel, W. Anderson, J. Eldridge, B. Glossop, and G. Willmott, Anal. Chem. 84, 3125 (2012).

[22] B. J. Sanghavi, W. Varhue, J. L. Chavez, C. Chou, and N. S. Swami, Anal. Chem. 86, 4120 (2014).

[23] K. D. Nyberg, M. B. Scott, S. L. Bruce, A. B. Gopinath, D. Bikos, T. G. Mason, J. W. Kim, H. S. Choi, and A. C. Rowat, Lab Chip 16, 3330 (2016).

[24] E. F. Hasselbrink, Jr., T. J. Shepodd, and J. E. Rehm, Anal. Chem. 74, 4913 (2002).

[25] W. A. Braff, A. Pignier, and C. R. Buie, Lab Chip 12, 1327 (2012).

[26] Y. Kazoe, K. Mawatari, and T. Kitamori, Anal. Chem. 87, 4087 (2015).

[27] K. Mawatari, Y. Kazoe, H. Shimizu, Y. Pihosh, and T. Kitamori, Anal. Chem. 86, 4068 (2014).

[28] H. Wu, Y. Chen, Q. Zhou, R. Wang, B. Xia, D. Ma, K. Luo, and Q. Liu, Anal. Chem. 88, 2502 (2016).

[29] A. McMullen, H. W. de Haan, J. X. Tang, and D. Stein, Nat. Commun. 5, 4171 (2014).

[30] K. E. Venta, M. B. Zanjani, X. Ye, G. Danda, C. B. Murray, J. R. Lukes, and M. Drndic, Nano Lett. 14, 5358 (2014)

[31] B. Chun and A. J. C. Ladd, Phys. Rev. E 75, 066705 (2007).

[32] B. Dünweg, U. D. Schiller, andA. J. C. Ladd, Phys. Rev. E 76, 036704 (2007).

[33] A. J. C. Ladd, R. Kekre, and J. E. Butler, Phys. Rev. E 80, 036704 (2009).

[34] J. D. Weeks, D. Chandler, and H. C. Anderson, J. Chem. Phys. 54, 5237 (1971). 